\title{
AVAliaÇÃo de tÉCNICA ELETROQUímica NA REMOÇÃo dE RESÍDUOS ORGÂNICOS EM ÁGUA COM EMPREGO DE UNIDADE EM ESCALA DE LABORATÓRIO
}

\section{EVALUATION OF ELECTROCHEMICAL TECHNIQUE FOR REMOVAL OF ORGANIC WASTE FROM WATER BY USING A LABORATORY SCALE UNIT}

\author{
ELIANDRO REZENDE DA SILVA \\ MsC em Engenharia de Materiais, bolsista, EMBRAPA Instrumentação Agropecuária
}

ODÍlIO BENEDITO GARRIDO ASSIS

Dr. em Engenharia pesquisador da EMBRAPA Instrumentação Agropecuária

Recebido: 16/03/04 Aceito: 27/07/04

\section{RESUMO}

Neste trabalho são apresentados os resultados preliminares da avaliação de técnica eletroquímica na remoção de compostos orgânicos em água, em escala laboratorial. Cela eletroquímica simples foi construída para os testes e uma metodologia básica foi empregada. O método consiste em provocar a migração de espécies polares ou ionizadas em direção ao eletrodo de carga oposta. Fenol e o herbicida atrazina foram os contaminantes avaliados. Os ensaios foram realizados em função do tempo de aplicação de carga, acompanhada a evolução do $\mathrm{pH}$ entre os eletrodos e as concentraçôes relativas avaliadas por medidas de absorbância na regiāo do ultra-violeta. Os melhores resultados foram conseguidos para operaçôes acima de 30 minutos, indicando migração proporcional a $62 \%$ para o fenol, mas inferior a $30 \%$ para a atrazina.

PALAVRAS-CHAVE: Remediação eletrocinética, tratamento eletroquímico de água, processo eletroquímico, fenol, atrazina.

\begin{abstract}
In this work the preliminary results of a lab scale evaluation of electrokinetic technique for the removal of organic contaminants in water are presented. A simple cell was constructed and a basic methodology was followed. The method consists in provoke the migration of ionized or polar specimens solved in aqueous solution in direction of the opposite charged electrode. Phenol $\left(\mathrm{C}_{6} \mathrm{H}_{5} \mathrm{OH}\right)$ and herbicide atrazine (6-chlorine- $N$-ethyl-N'-(1-methylethyl)1,3,5-triazine-2,4 diamine), was chosen as standard contaminants. The testes were performed in function of charge application time, mapping the $p H$ between electrodes and the concentration assessed by absorbance measurements in ultra-violet region. The best results were attained after 30 minutes operation, showing migration proportional to $62 \%$ for phenol, but inferior to $30 \%$ atrazine.
\end{abstract}

KEYWORDS: Electrokinetic remediation, water purification, electrochemical process, phenol, atrazine.

\section{INTRODUÇÃO}

Técnicas baseadas em reações eletroquímicas e suas variantes têm sido objeto de avaliações contínuas em processos de remediação ambiental, com resultados promissores na purificação de águas residuais e, em particular, na remoção de metais pesados em meios porosos (Hicks \& Tondorf, 1994; Acar et al., 1995). Esses processos têm como princípio a migração de espécies, sob a ação de um campo elétrico gerado por meio de eletrodos, provocando no sistema uma camada difusa móvel que carrega com ela os compostos polares presentes em solução. $\mathrm{Na}$ presença de meios com superfícies ou partículas, como solos, estes são considerados fixos com relação ao movimento da camada, sendo a este fenômeno dado o nome de eletroosmose (Crockford, 1977).

Casagrande, em 1949, foi um dos pioneiros na avaliação do transporte de água em meios porosos através de técnicas eletroquímicas, estabelecendo os primeiros fundamentos do processo. A partir da década de 1960, demais mecanismos que faziam parte do fenômeno eletrocinético, mas menos compreendidos, foram melhor avaliados e conseqüentemente considerados na conceituação da eletroosmose. Os modelamentos de hoje são abrangentes e levam em consideração diversos fatores como simultaneamente atuantes no processo eletroquímico (Alshawabkeh \& Acar, 1996).

Em princípio, as técnicas eletrocinéticas são passíveis de aplicação em qualquer meio permeável humidificado, onde o contaminante a ser removido, é do tipo inorgânico, orgânico ou organometálico, adsorvidos ou não sob superfícies sólidas, ou de espécies carregadas (iônicas) e não carregadas (polar e não polar) em solução (Denisov, et al., 1996).

Técnicas baseadas no princípio de eletroosmose tem sido atraente do ponto de vista prático principalmente em processos de geotecnologia, como na drenagem e estabilização de solos de textura fina (Segall, et al., 1980; Acar, \& Alshawabkeh, 1993). Dentre as aplicaçōes com sucesso na remoção de contaminantes, pode-se citar a migração de ions $\mathrm{Cu}^{2+}$, $\mathrm{Cd}(\mathrm{II})$ e $\mathrm{Pb}$ (II) de águas e solos contaminados pela indústria de baterias (Hamed, et al., 1991; Viadero, etal., 1998), 
na remoção de hidro-carbonetos em solos (Bruell, et. al., 1992) e na extração de fenol em kaolinita (Acar et al., em 1992) entre outras. Recentemente, foi demonstrado a aplicabilidade da técnica de eletroosmose na recuperação de filtros cerâmicos contaminados por agentes químicos (Assis e Silva, 2003).

Neste trabalho foi avaliado o emprego da técnica eletrocinética na remoção isolada de resíduos orgânicos em água em escala de laboratório. O principal objetivo foi o de estabelecer uma metodologia mínima para aferir a eficiência da técnica na migração de resíduos de agrotóxicos em meio aquoso.

Dois contaminantes, fenol e atrazina, foram avaliados. $\mathrm{O}$ fenol foi escolhido como um elemento teste, não somente por sua toxicidade, mas igualmente por apresentar uma alta solubilidade em água e ser um dos principais resíduos das refinarias de óleo, indústrias de plástico, tintas e pesticidas. O agrotóxico atrazina, é um herbicida amplamente empregado no Brasil no controle geral de ervas daninhas, principalmente nas plantações de cana de açúcar e milho. Faz parte do grupo das striazinas que são compostos de alta mobilidade e persistência, além de danosos à saúde humana.

\section{METODOLOGIA}

\section{Concentração dos contaminantes}

Inicialmente soluçôes estoques com contaminantes (fenol eatrazina a $6 \mathrm{mg} / \mathrm{ml}$ ), foram separadamente preparadas por dissolução em água destilada. Diluições a partir dessa concentração foram realizadas, levantando-se assim curvas de calibração, tendo por referência medidas de absorbâncias obtidas por espectroscopia de UV-Vis (equipamento Shimadzu, UV-1601PC), tomando as intensidades medidas no comprimento de onda $270 \mathrm{~nm}$ para o fenol e em $223 \mathrm{~nm}$ para a atrazina (Silva e Assis, 2002). Com base nestas correspondências torna-se possível estabelecer relações de proporções numéricas das concentrações resultantes do ensaio eletroquímico.

\section{Processo eletroquímico}

Para a realização dos experimentos uma cela eletroquímica simples foi confeccionada em acrílico com paredes de $0,8 \mathrm{~cm}$ de espessura, para um volume de $700 \mathrm{~cm}^{3}$. Empregou-se eletrodos planos de titânio com área de trabalho igual a $26 \mathrm{~cm}^{2}$, paralelamente alocados na cela, conforme disposição apresentada no esquema da Figura 1. Os testes foram realizados sob tensão de $60 \mathrm{~V}$ (DC) e corrente constante de 5,0 $\mathrm{mA}$ aplicadas diretamente nos eletrodos. Detalhes sobre a confecção da cela e da montagem experimental podem ser encontrados em Silva, 2001.

Os testes foram realizados isoladamente em cada solução contaminada. A adição do fenol se deu na concentração de $1 \mathrm{mg} / \mathrm{ml}$ (empregado-se fenol comercial, Synth - lote 15138). Após homogeneização o pH medido foi de 6,0. $\mathrm{O}$ pH inicial da água destilada era de 5,56.

Para a contaminação com atrazina (6-cloro-N-etil-N'-(1-metiletil)-1,3,5triazina-2,4 diamina), uma segunda solução foi elaborada, também a partir de produto de origem comercial (Primestra Gold, Syngenta) a uma concentração de $2,0 \mathrm{mg} / \mathrm{ml}$. O pH da solução de atrazina foi medido em 5,82. O aumento do $\mathrm{pH}$ para ambos contaminantes indica ionização das moléculas na presença de água, o que torna apropriado ao processo eletroquímico.

Todos os experimentos foram realizados na temperatura ambiente. A evolução do processo foi acompanhada pela varredura do $\mathrm{pH}$ ao longo do comprimento da célula tomados do catodo para o anodo para diversos tempos de operação (em intervalos de 30 minutos) e a análise da densidade ótica do meio líquido nas regiões próximas aos eletrodos, por espectrofotometria de UV-Vis, tendo por referência de concentração as curvas padrão previamente levantadas. A medida do $\mathrm{pH}$ acusa a geração de gradiente de potencial químico, como combinação entre a eletrólise da água e o fluxo eletroquímico, no qual efetuará a migra-
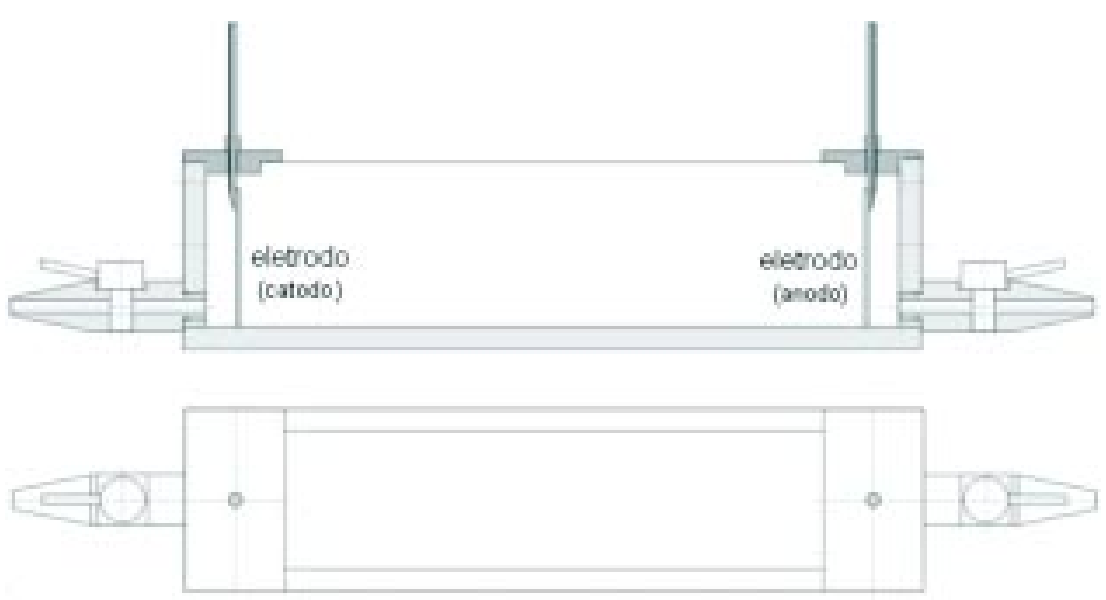

Figura I- Vista lateral e superior da célula eletroquímica utilizada ção dos contaminantes. Todas as medidas foram realizadas em triplicatas.

\section{RESULTADOS E DISCUSSÃO}

\section{Migração de fenol}

$\mathrm{Na}$ Figura 2 temos o perfil de $\mathrm{pH}$ tomado ao longo do comprimento da célula para a solução contaminada com fenol. De um modo geral, observa-se um comportamento similar para todos os tempos medidos, com um aumento gradual da acidez na direção do anodo. Este efeito é esperado e indica o processo eletroquímico com o estabelecimento de concentraçôes básicas no catodo e ácidas no anodo, estabelecidas principalmente por mecanismos de eletrólise.

Com a manutenção da tensão a migração dos ions hidrogênios é favorecida em direção ao anodo e dos grupos hidroxilas para o catodo, arrastando assim o fenol em solução. Para períodos superiores a 30 minutos tem-se uma queda significativa do $\mathrm{pH}$ a partir do catodo, com estabilização em torno de 4,5 próximo ao anodo. Essa queda para valores abaixo do $\mathrm{pH}$ inicial, medido em 6,0, não pode ser interpretada apenas como resultado da migração do fenol, mas também por processos simultâneos de eletrólise decorrente da carga aplicada. Segundo Acar et al., 1995, o processo eletroquímico promove a movimentação de produtos da eletrólise da água $\left(\mathrm{OH}^{-} \mathrm{e}\right.$ $\mathrm{H}^{+}$) estabelecendo um fluxo de moléculas influenciadas pelos gradientes de potencial elétrico, hidráulico e químico. Como a molécula de fenol $\left(\mathrm{C}_{6} \mathrm{H}_{5} \mathrm{OH}\right)$ apresenta alta solubilidade em água devido a presença de grupos hidroxilas, sua inserção no meio fará com que os fenóis 
com seus grupos $\mathrm{OH}^{-}$tomem parte da migração dos grupos $\mathrm{OH}^{-}$resultantes do processo eletrolítico da água.

O efeito da migração pode ser melhor visualizado comparando, por exemplo, os espectros de absorção do fenol, realizadas em amostras coletadas na região dos eletrodos. Na Figura 3 temos ilustrado as intensidades obtidas inicialmente e após os 90 minutos de aplicação da carga em amostra recolhida próxima ao anodo. Os espectros indicam uma redução significativa das intensidades da absorção em seu comprimento de onda típico, próximo a $240-270 \mathrm{~nm}$.

De uma forma geral esses resultados confirmam o processo de drenagem das moléculas de fenol junto com grupos hidroxilas em direção ao catodo devido a polaridade do campo. Com base na curva de calibração, os resultados de absorvância indicam que após as aplicaçôes de correntes por 90 minutos, ocorre uma redução proporcional de $62 \%$ da quantidade de fenol na região do anodo com acréscimo de concentração proporcional no catodo. A migração só não é total devido a fatores como a oxidação e adsorção do fenol no eletrodo, como também a formação de outras espécies de alta solubilidade em água (Ferreira, 2000), reduzindo a eficiência do processo e conduzindo a uma estabilização dos parâmetros medidos.

\section{Migração do herbicida atrazina}

De forma similar, a Figura 4 apresenta os resultados da variação do $\mathrm{pH}$ em função do tempo, tomados ao longo do comprimento da célula para a reação na soluçáo contaminada com o herbicida atrazina, indicando comportamento parecido ao do fenol, embora menos acentuado.

$\mathrm{O}$ fenol e a atrazina possuem estruturas similares, caracterizados por nitrogenados heterocíclicos (estrutura de anel). A molécula da atrazina pode sofrer hidrólise dando origem ao metabólito hidroxiatrazina, que é igualmente um composto tóxico (Armstrong \& Chesters, 1968). De uma forma geral, vemos que a eletromobilidade da atrazina é bem inferior à observada para o fenol, o que traduz em uma menor eficiência de migração no meio aquoso, o que pode ser comprovado pelas intensidades das absorvâncias medidas no anodo, Figura 5 , correspondendo a uma eficiência da ordem de $28 \%$, bem inferior aos valores obtidos para o fenol.

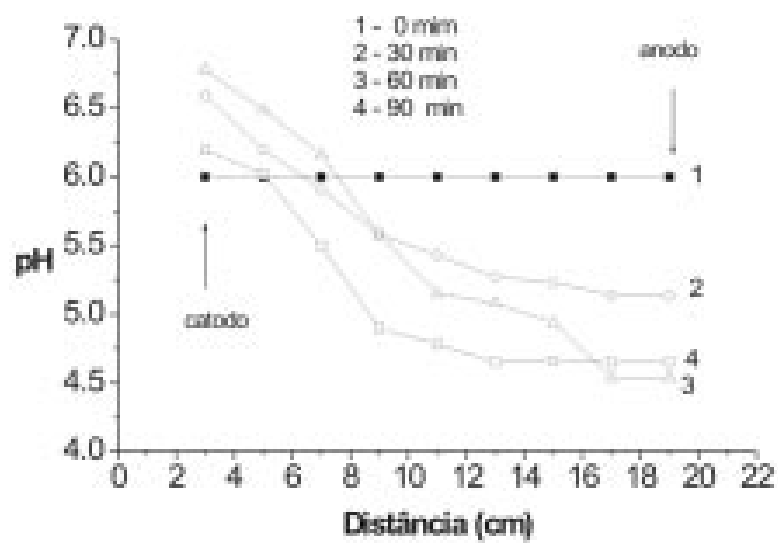

Figura 2 - Variação do pH ao longo da célula para tempos crescentes de aplicação de operação (contaminante fenol)

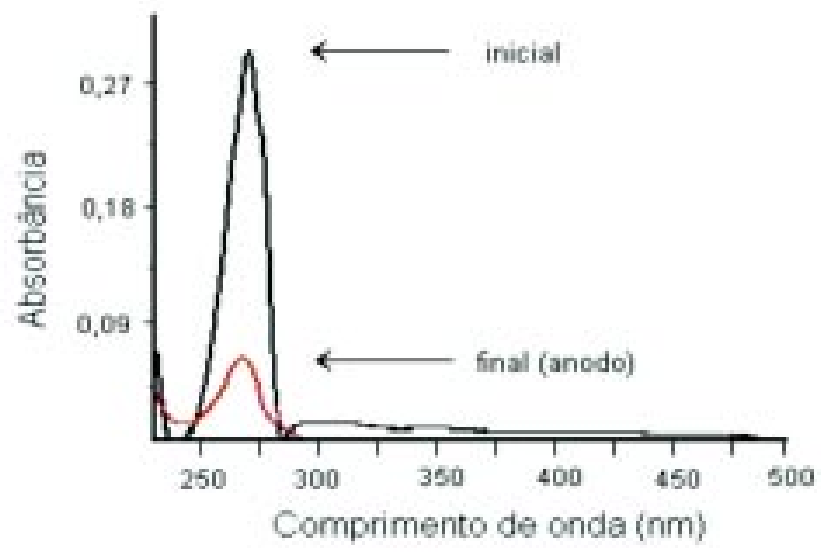

Figura 3 - Espectros de UV-Vis para amostras coletadas na região do anodo, no início e após 90 minutos de experimento (contaminante fenol)

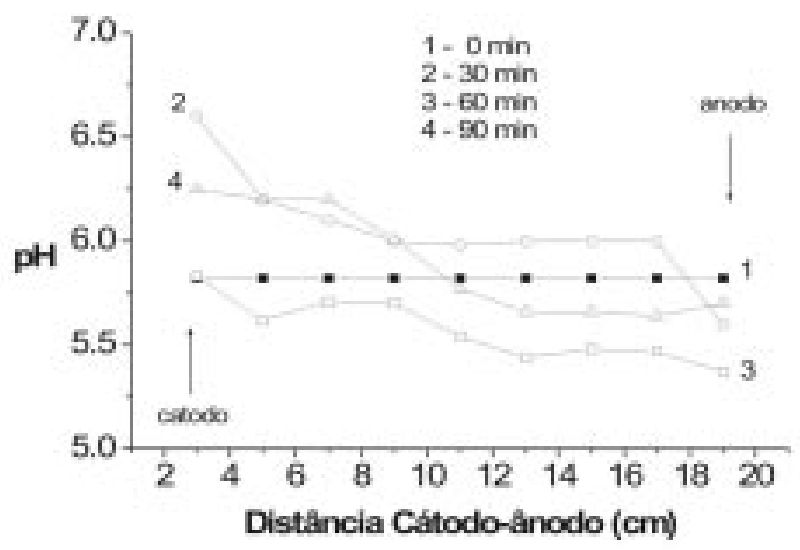

Figura 4 - Variação do pH ao longo da célula para tempos crescentes de aplicação de operação (contaminante atrazina) 


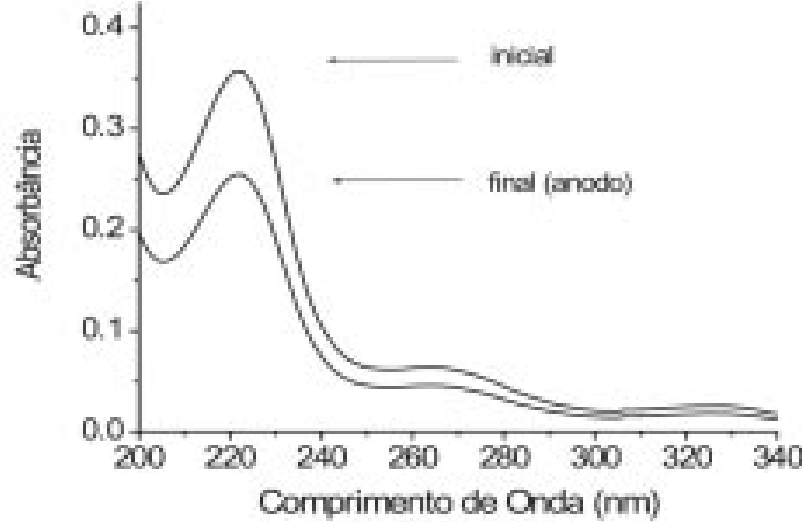

Figura 5 - Espectros de UV-Vis para amostras coletadas na região do anodo, no início e após $\mathbf{9 0}$ minutos de experimento (contaminante atrazina)

\section{CONCLUSÕES}

O objetivo principal deste trabalho foi avaliar algumas condiçōes experimentais que possam vir a ser empregadas na prática para a remediação eletroquímica de compostos orgânicos presentes em água.

Nas condições de laboratório aqui apresentadas, confirmou-se a possibilidade da migração e concentração de contaminantes em polaridade determinada, tornando possível sua posterior remoção. As melhores eficiências foram obtidas para períodos superiores a 30 minutos de operação, indicado pela concentração dos contaminantes na região do catodo. As análises complementares por absorçãoo de UV indicam eficiência superior a $60 \%$ na migração do fenol e próxima a $30 \%$ para a atrazina. A larga diferença entre os valores obtidos indica que o processo é seletivo sendo necessário a aplicação de tensōes distintas para a migração satis- fatória de contaminantes distintos.

Contudo, de uma forma geral, os resultados confirmam o potencial da técnica, onde através de montagens simples e práticas, possa ser satisfatoriamente empregada como procedimento coadjuvante e de baixo custo na purificação de águas contaminadas por compostos químicos

\section{AGRADECIMENTOS}

Os autores são gratos ao $\mathrm{CNPq}$ e EMBRAPA por recursos disponibilizados.

\section{REFERÊNCIAS}

ACAR, Y. B.; GALE, R. J.; ALSHAWABKEH, A. N. Electrokinetic Remediation: Basic Principles and Technology Satus. J. of Hazardous Materials. v. 40, p.117-137, 1995.

ACAR, Y. B.; ALSHAWABKEH, A. N Principles of Electrokinetic Remediation. Environmental Science Technology. v.27, p.2638-2647, 1993.

ACAR, Y. B., LI, H.; GALE, R. J. Phenol Removal from Kaolinite by Electrokinetics. Journal of Geotechnical Engineering ASCE. v. 118, n.11, pp. 1837-1852 1992.

ALSHAWABKEH, A. N.; ACAR, Y. B. Electrokinetic Remediation II: Theoretical Model. J. Geotechnical Engineering. v. 122, n.3 p.186-196, 1996.

ARMSTRONG, D.E.; CHESTERS, G. Adsorption catalyzed chemical hydrolysis of atrazine. Environmental Science and Technology , v. 9 p.683-689, 1968.

ASSIS, O. B. G., SILVA, E. R. The Use of Electrochemical Technique For Ceramic Filtration Medium Cleaning. In: FOURTH INTERNATIONAL LATINAMERICAN CONFERENCE ON POWDER TECHNOLOGY, 2003, Guarujá, SP. Proccedings of PTECH 2003 - CD ROM - paper 17/15. Associação Brasileira de Cerâmica, 6p., 2003.

BRUELL, C. J.; SEGALL, B. A.; WALSH, H. T. Electroosmotic Removal of Gasoline Hydrocarbons and TCE from Clay. J. of Environmental Engineering. v.118, n.1 p.6883, 1992.

CASAGRANDE, I. L. Electro-Osmosis in soils. Geotechnique. v.1, p.1959-1977, 1949.

CROCKFORD, H. D. Fundamentos de físicoquímica. 1. ed. Rio de Janeiro: LTC. 383p., 1977.

DENISOV, G.; HICKS, R. E.; PROBSTEIN, R. F. On the Kinetics of Charged Contaminant Removal from Soils Using Electric Fields. J. of Colloid and Interface Science. v.178, p.309323, 1996.

FERREIRA, M. Estudos da Oxidação Eletroquímica de Substâncias Fenólicas. Tese (Doutorado). Instituto de Química de São Carlos, Universidade de São Paulo. São Carlos. 131p., 2000.

HAMED, J. T.; ACAR, Y. B.; GALE, R. W. Pb II Removal from Kaolinite by Electrokinetics.
Journal of Geotechnical Engineering, v.2, n.117 p.241-271, 1991

HICKS, R. E, TONDORF, S. Electrorestoration of metal contaminated soils. Environmental Science and Technology v. 28, n.12 p.22032008, 1994.

SEGALL, B. A.; O’BANNON, C. E.; MATTHIAS, J. A. Electro-Osmosis Chemistry and Water Quality. Journal of Geotechnical Engineering ASCE. v.106, n.GT10 p.11481152,1980

SILVA, E. R., ASSIS, O. B. G. Eletroosmose Aplicada na Remediação química de meios particulados. Anais da Associação Brasileira de Química. v.51, n.2 p.53 - 57, 2002.

SILVA, E. R. Uso de Técnicas de Eletroosmose para Purificação de Sistemas Particulados. Mestrado em Ciência e Engenharia de Materiais, Universidade de São Paulo, São Carlos, 89p. 2001

VIADERO, R. C. J. et al. A Laboratory-Scale Study of applied Voltage on the electrokinetic Separation of Lead from Soils. Separation Science and Technology. v.12, n.33 p.1833-1859, 1998.

Endereço para correspondência:

Odílio Benedito Garrido Assis

EMBRAPA Instrumentação

Agropecuária

Rua XV de Novembro, 1452

I3560-970 - São Carlos - SP - Brasil

Tel.: (I6) 2742477

Fax: (I6) 2725958

Email:odilio@cnpdia.embrapa.br 